\title{
Comparison of Peripheral Arterial Blood Pressure in Individuals with and without Forward Head Posture
}

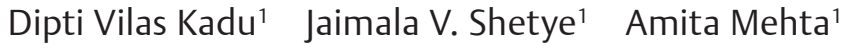 \\ ${ }^{1}$ Department of Physiotherapy, Seth G. S. Medical College and \\ K.E.M. Hospital, Parel, Mumbai, Maharashtra, India \\ Address for correspondence Dipti Vilas Kadu, MPTh, Department of \\ Physiotherapy, Seth G. S. Medical College and K.E.M. Hospital, Parel, \\ Mumbai 400012, Maharashtra, India (e-mail: kadu_dipti@yahoo.com).
}

J Health Allied Sci NU 2019;9:98-103

\begin{abstract}
Introduction Cervical spine is closely approximated with neurovascular structures. Therefore, misalignment of cervical spine so commonly seen today may lead to altered blood pressure (BP). There could be a relationship between head neck posture as measured by craniovertebral angle and peripheral arterial BP.

Aim The aim of this study is to compare peripheral arterial BP in individuals with and without forward head posture (FHP).

Study Design This is a comparative, prospective, observational study in healthy population.

Subjects and Methods A total of 150 students were selected randomly and screened so that there were equal and desired number of subjects in the two groups (64 in each group) for this comparative, prospective, observational study. Three readings of BP were taken in sitting position on brachial artery at interval of 2 to 3 minutes. One standard image was taken in lateral view used for measuring craniovertebral angle with "MB ruler software." Craniovertebral angle, systolic blood pressure (SBP), and diastolic blood pressure (DBP) of the two groups were compared.

Keywords

- forward head posture

- blood pressure

- hypertension

- neurovascular compression

- physiotherapy

Results The aim of the study was to compare peripheral arterial BP in individuals with and without FHP. The mean SBP and DBP of both the groups were within the normal range as was expected because the subjects were young students with no clinical symptoms. However, it was seen that the mean SBP was significantly higher in subjects with FHP than in subjects without FHP $(p=0.0009)$.

Conclusion Peripheral arterial BP in individuals with FHP is statistically significantly higher than in individuals without FHP.
\end{abstract}

\section{Introduction}

Computers have become an integral part of today's lifestyle. Increasing computer literacy is advocated by the government and it is indeed necessary for progress. However, its overuse for profession or for recreation has cast its effect on the human body, one of it being a forward head posture (FHP), a most common postural issue.

FHP is characterized by increased flexion of lower cervical and upper thoracic regions, increased extension of occiput on the first cervical vertebra, and increased extension of the upper cervical vertebrae. ${ }^{1}$ Today, its prevalence in America is $66 \%{ }^{2}$ The common causes of FHP are effects of gravity, slouching, poor ergonomic alignment in the work or home environment, postures requiring leaning forward or tipping the head backward for extended periods, and computer workstations not ergonomically specific to the user. ${ }^{1}$

"Craniovertebral angle" (CV angle) is simple and convenient clinical descriptor of natural head position. ${ }^{3} \mathrm{CV}$ angle is formed by the intersection of the horizontal line passing through $C_{7}$ and a line extending from tragus of the ear to $C_{7}$ on an image. ${ }^{4}$ This technique can be applied reliably to measure natural head neck posture in the sagittal plane (intraclass correlation coefficient $=0.98)^{3}$ received

August 28, 2019

accepted after revision

October 21, 2019
DOI https://doi.org/

10.1055/s-0039-3400686.

ISSN 2582-4287.
Copyright $\odot 2019$ Nitte University

(Deemed to be University)
License terms

(ㅇ) (1) $\ominus \circledast$ 
Hypertension in adults is defined as systolic blood pressure (SBP) of $\geq 140 \mathrm{~mm} \mathrm{Hg}$ and/or diastolic blood pressure (DBP) of $\geq 90 \mathrm{~mm} \mathrm{Hg}$ or any level of BP in patients taking antihypertensive medication. ${ }^{5}$ Recent reports indicate that nearly 1 billion adults had hypertension in 2000 and this is predicted to increase up to 1.56 billion by 2025 . Earlier reports also suggest that the prevalence of hypertension is rapidly increasing in developing countries and is one of the leading causes of death and disability. ${ }^{5}$

At first, we may not grasp a relationship between spine and cardiovascular function. The spine protects the spinal cord that is main connection between the brain and the body. The central nervous system specifically at the level of $C_{1}$ controls many of the body systems and functions. Therefore, research has been increasingly focusing on the link between biomechanical disorders of spine and the effect that it has on nervous system function. Though there are many factors that contribute to hypertension that are mainly lifestyle related, the role of the nervous system in the regulation of blood pressure cannot be ignored. ${ }^{6}$

As per the pilot study by Bakris et al, restoration of atlas alignment is associated with marked and sustained reduction in BP similar to use of two drug combination therapy to control BP. This study had documented that atlas vertebra realignment helped to reduce arterial pressure in hypertensive patients. ${ }^{7}$ We hypothesized that:

1. Malalignment of the cervical spine at the level of the atlas vertebra is associated with relative ischemia of the brainstem circulation and increased BP and

2. Change in the anatomical position of the atlas and resultant change in the vertebral artery lends itself to worsening of hypertension.

This study was conducted to assess the relationship between peripheral arterial BP and head neck posture as measured by CV angle.

\section{Subjects and Methods}

Subjects: Based on the standard deviations in pilot study, sample size calculated was 127 . It was decided that there would be 64 subjects in each group. A total of 150 students were selected randomly from "Physiotherapy and Occupational Therapy Department" between age group 18 to 30 years who were willing to participate in the study. The selection of extra subjects was done with a view of getting 64 subjects with FHP, which we assumed to be difficult to find as most of them would be without FHP as they all were asymptomatic healthy individuals as described in inclusion criteria. The subjects were screened till each group received 64 subjects. Subjects having current or past history of cardiovascular and respiratory illness, high body mass index $\left(\geq 25 \mathrm{~kg} / \mathrm{m}^{2}\right),{ }^{8}$ and smoking were excluded from study.

Ethical approval and informed consent: The study was approved by the Ethics Committee of the institute and university. The subjects were admitted to the study after they signed the written informed consent document.
Procedure to measure BP: Peripheral arterial BP was measured on brachial artery by auscultatory method in sitting position. ${ }^{9}$ Three readings of SBP and DBP were recorded at interval of 2 to 3 minutes. Average of three readings that is mean SBP and mean DBP was considered. ${ }^{5}$

Procedure for marking $C 7$ spinous process and tragus of ear: $C_{6}$ and $C_{7}$ spinous processes are most obvious. $C_{6}$ and $C_{7}$ were differentiated by passively flexing and extending the subject's neck. With this movement, the $C_{6}$ spinous process moves in and out, and the $C_{7}$ spinous process remains stationary. ${ }^{6}$ By this method, $C_{7}$ spinous process was identified by palpation. $C_{7}$ spinous process and tragus of the ear were marked with body surface sticker.

Procedure for taking image: For the photographic session, subjects wore a collarless dress. ${ }^{3}$ All subjects were instructed to sit comfortably on a high back chair with both feet flat on the floor, hips, and knees positioned at 90 and buttocks positioned against the back of the chair. They were requested to sit as usually they sit and keep their both hands on lap. ${ }^{4}$ Subjects were instructed to fix their gaze on the reference point in the eye-line on the wall facing the subject. ${ }^{3}$ Images were taken using COOLPIX S6300 camera with 50\% zooming power and flash on. Camera was supported on tripod. The height (distance between lens of the camera and ground) was $110 \mathrm{~cm}$ and distance (distance between lens of the camera to subject's tragus of the ear) was $150 \mathrm{~cm}$. The height of the camera from ground, distance of the camera from the subject, and focus-alignment-orientation of the camera were standardized and ensured for every shoot. ${ }^{3}$ The camera was positioned so that all anatomical markers were detectable in one single image. ${ }^{10}$ One standard image was taken in sitting position in lateral view to assess FHP objectively with the help of CV angle. ${ }^{4}$ Images provide valid and reliable indicators of the position of the underlying spine in sitting. ${ }^{10}$

$\mathrm{CV}$ angle measurement: Once the image was obtained, it was used to measure the CV angle. " $\mathrm{CV}$ angle" is the angle between the horizontal line passing through $C_{7}$ and a line extending from tragus of the ear to $C_{7}$. A smaller CV angle indicates a greater FHP., ${ }^{411}$ The images were transferred to the computer via USB data transfer cable. ${ }^{10} \mathrm{CV}$ angle was measured by using “MB-Ruler 5.0 Software.” The Cronbach's $\alpha$ coefficient value for intra-rater $(0.999)$ as well as interrater (0.892) reliability is very high, thus "MB Ruler Software" is reliable for measuring CV angle.

Average $\mathrm{CV}$ angle is $50^{\circ}$ in pain-free population. $\mathrm{CV}$ angle $<50^{\circ}$ is associated with neck pain, tension headaches, and reduced cervical range of motion. ${ }^{11}$

Depending on CV angle, subject was placed into Group A and Group B. ${ }^{11}$

- Group A: Subjects with FHP, that is, CV angle $\leq 50$.

- Group B: Subjects without FHP, that is, CV angle $>50$.

Total 150 subjects were screened so that there would be 64 subjects in both the groups.

Outcome measures: CV angle and BP (SBP and DBP). 


\section{Results}

Data was analyzed using Graph-Pad Instat Version 3. Data normality of the data was tested by using Kolmogorov-Smirnov test. As the data did not follow normal distribution, for parameters SBP, DBP nonparametric test was used to analyze these parameters. Mann-Whitney $U$-test was applied to compare their mean. The statistical significance was set at $p<0.05$.

The aim of this study was to compare peripheral arterial BP in individuals with and without FHP. The mean SBP and DBP of both the groups were within the normal range as was expected because the subjects were young students (-Tables 1 and $\mathbf{2}$ ) with no clinical symptoms. However, it was seen that the mean SBP was significantly higher in subjects with FHP than in subjects without FHP (-Table 3 )

(-Figs. 1 and 2 ).

Another striking finding was that out of 64 FHP subjects, 23 subjects were in the prehypertension range (-Table 4 ). However, the other group had only 6 subjects with BP in that range. None of the subjects in any group had hypertension (-Table 5).

\section{Discussion}

The upper cervical region consists of first and second cervical vertebrae $\left(C_{1}\right.$ and $\left.C_{2}\right)$ and lower cervical region consist of third to seventh cervical vertebrae $\left(C_{3}\right.$ to $\left.C_{7}\right)$. ${ }^{6,12,13}$ Two concave superior zygapophyseal facets of the atlas articulates with two convex occipital condyles of the skull to form

Table 1 Age distribution

\begin{tabular}{|l|l|l|}
\hline & Group A & Group B \\
\hline Mean (years) & 21.21 & 21.29 \\
\hline Standard deviation & 2.29 & 1.85 \\
\hline
\end{tabular}

Table 2 Gender distribution (numbers)

\begin{tabular}{|l|l|l|}
\hline & Group A & Group B \\
\hline Female & 49 & 49 \\
\hline Male & 15 & 15 \\
\hline
\end{tabular}

atlanto-occipital joints. In two atlantoaxial joints, the articular cartilages of both the atlantal and axial facets are convex, rendering the zygapophyseal facet joints biconvex. In FHP, occipital condyles roll backward and slide forward due to extension of upper cervical spine., ${ }^{1,12}$ This suboccipital joint has maximum mobility and mechanical activity, compared with other intervertebral joints. ${ }^{13}$ The atlas is more prone to malalignment, as it depends solely upon soft tissues like muscles and ligaments to sustain its alignment, unlike other vertebrae that interlock one to the next. ${ }^{7}$ When these upper cervical vertebrae, which are located next to the skull, are misaligned, they can restrict arterial circulation and nerve flow that affects many bodily functions including BP. ${ }^{7}$

\section{Neural Mechanism}

The central nervous system is protected by the cranium and the vertebral canal. The cervical column protects the lower medulla oblongata as it emerges through the foramen magnum and the cervical spinal cord. Thus, the medulla and the cervical cord are closely related to the highly mobile parts of the cervical column, especially in the suboccipital region that is the highly specialized zone of mechanical transition. In fact, as the medulla emerges through the foramen magnum to become the spinal cord, it lies behind and between the two occipital condyles that allow the skull to rest on the cervical column. ${ }^{13}$ Therefore, chronic malalignment of cervical vertebrae like FHP may lead to harmful effects on various body functions including BP.

As the cervical spine is closely related to the neural structures, and the nervous system has a substantial role in maintaining the BP, anatomical malalignments of the cervical vertebrae especially at the atlas vertebra might result into relative ischemia of brainstem circulation, which can lead to increased BP. Even with minor misalignment of atlas vertebra, there are chances of injury, impairment, and/or compression of neural pathways of brainstem. The correlation between hypertension and circulatory abnormalities in the area around the atlas vertebra and posterior fossa of the brain has been proven. ${ }^{7.14}$

As per the study by Reis et al in rats, the neurons of the lower brainstem, located in the ventrolateral medulla, are accountable for controlling normal levels of arterial pressure.

Table 3 Statistical analysis

\begin{tabular}{|l|l|l|l|}
\hline & Group & SBP $(\mathrm{mm} \mathrm{Hg})$ & DBP $(\mathrm{mm} \mathrm{Hg})$ \\
\hline Mean & A & $114.13(\mathrm{SD} \pm 10.83)$ & $74.65(\mathrm{SD} \pm 8.80)$ \\
\hline & B & $107.64(\mathrm{SD} \pm 8.06)$ & $70.37(\mathrm{SD} \pm 6.15)$ \\
\hline Difference & & 6.5 & 4 \\
\hline Minimum & A & 95.33 & 56.33 \\
\hline & B & 91.33 & 52.33 \\
\hline Maximum & A & 136 & 101.33 \\
\hline & B & 123.33 & 87.33 \\
\hline p-value & & 0.0009 & 0.0023 \\
\hline
\end{tabular}

Abbreviations: DBP, diastolic blood pressure; SBP, systolic blood pressure; SD, standard deviation. 


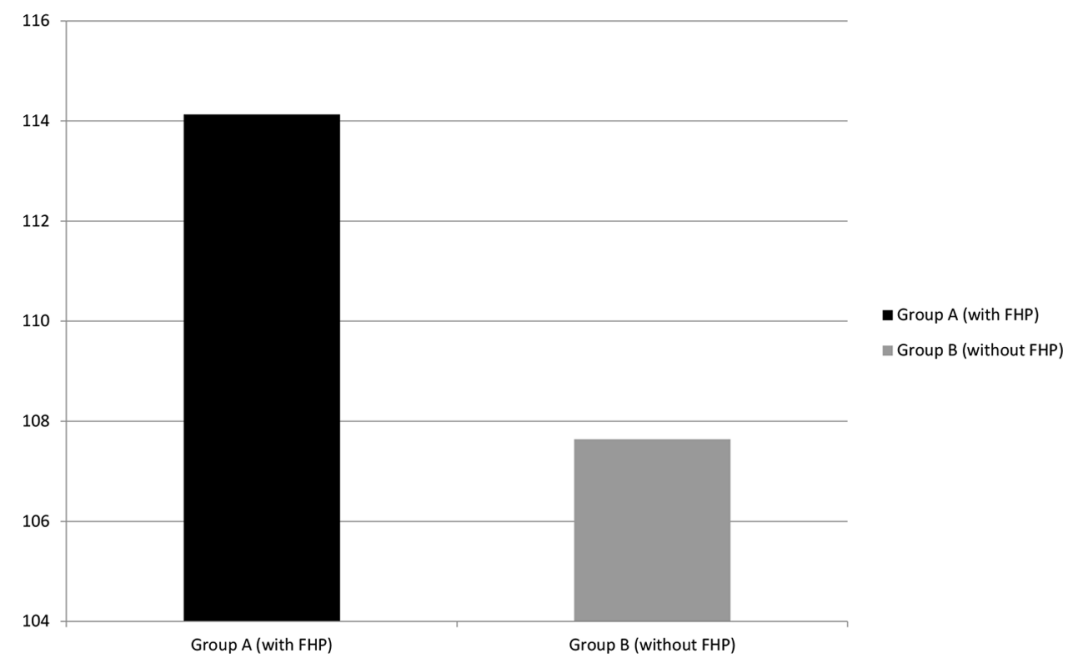

Fig. 1 Systolic blood pressure. FHP, forward head posture.

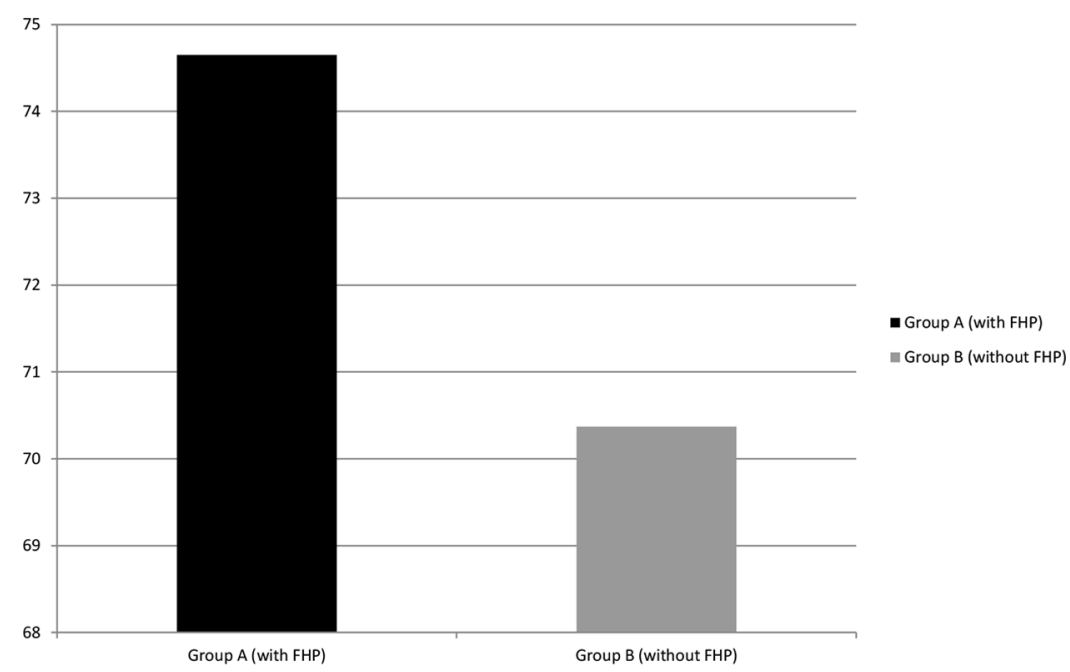

Fig. 2 Diastolic blood pressure. FHP, forward head posture.

Table 4 Classification of BP for adults ${ }^{9}$

\begin{tabular}{|l|l|l|}
\hline BP classification & SBP $(\mathrm{mm} \mathrm{Hg})$ & DBP $(\mathrm{mm} \mathrm{Hg})$ \\
\hline Normal & $<120$ & and $<80$ \\
\hline Prehypertension & $120-139$ & or $80-89$ \\
\hline Stage 1 hypertension & $140-159$ & or $90-99$ \\
\hline Stage 2 hypertension & $\geq 160$ & or $\geq 100$ \\
\hline
\end{tabular}

Abbreviations: BP, blood pressure; DBP, diastolic blood pressure; SBP, systolic blood pressure.

Neurons of the $\mathrm{C}_{1}$ area have significant effect on normal resting and reflex control of arterial pressure. They may have important role in the control of increased arterial pressure in hypertension. They also act as site of action of antihypertensive drugs. ${ }^{15}$

Study of magnetic resonance imaging (MRI) done by Coffee et al showed an important correlation between arterial compression of the ventrolateral medulla and existence of hypertension. They proposed that MRI should be done in subjects
Table 5 Statistics according to blood pressure

\begin{tabular}{|l|l|l|}
\hline & Group A (with FHP) & $\begin{array}{l}\text { Group B } \\
\text { (without FHP) }\end{array}$ \\
\hline Normal & $41($ mean $107 \mathrm{~mm} \mathrm{Hg})$ & 58 (mean $106 \mathrm{~mm} \mathrm{Hg})$ \\
\hline $\begin{array}{l}\text { Prehyper- } \\
\text { tension }\end{array}$ & $23($ mean $126 \mathrm{~mm} \mathrm{Hg})$ & 6 (mean $122 \mathrm{~mm} \mathrm{Hg})$ \\
\hline Stage 1 & 0 & 0 \\
\hline Stage 2 & 0 & 0 \\
\hline
\end{tabular}

Abbreviation: FHP, forward head posture.

with increased BP to evaluate their posterior fossa for confirmation of any anatomical deviation. ${ }^{16}$

The conclusion of the study done by Jannetta et al suggested that, compression of the medulla oblongata and cranial nerves (CN IX and CN X) located near it, may affect the neurogenic regulation of BP. They strongly suggested that certain forms of hypertension are due to compression of the medulla oblongata and that can be reduced by decompression of the same. ${ }^{17}$ 
Study done by Akimura et al suggested that, blockade of cardiac C-fibers of the vagus nerve due to neurovascular compression (NVC), may lead to increase in BP. The medulla has vital role in cardiovascular control. The NVC at the medulla can result in permanent irritation and activation of the vasopressor neurons. ${ }^{18}$

As per the study done by Schobel et al, in case of essential hypertension (EHTN) NVC at ventrolateral medulla can be associated with raised central sympathetic activity. They also found that sympathetic vasoconstrictor outflow to skeletal muscles was distinctly raised in patients having EHTN with NVC, as compared with those without NVC and this can be correlated with systolic arterial pressure. From this study, neurological background for EHTN has been suggested for a subgroup of EHTN patients having chronic increase in sympathetic activity. ${ }^{19}$ Also, the malalignment or injury to cervical area may lead to hypertonia of sympathetic nervous system, as this area is closely related to brainstem. ${ }^{6}$

\section{Vascular Mechanism}

The vertebral arteries run upward on both sides of the cervical spine, around atlas and into the cranium. These arteries pass through the transverse processes of the cervical vertebrae. The vertebral arteries accompanying with the internal carotid artery fulfill $20 \%$ of the blood requirement of the brain, especially hindbrain. ${ }^{6}$ The suboccipital area has maximum mobility and mechanical activity as compared with other intervertebral joints of the vertebral column, as the atlas does not have facets like the rest of the vertebrae. ${ }^{13}$ The angiogram of cervical region shows a "bow-tie" appearance where the vertebral arteries wrap around the atlas vertebra before ascending to the cranium. From this, we can conclude that a chronic malpositioning of atlas vertebra like in FHP would cause disturbance in blood flow to the brainstem. Alterations in the circulation of the vertebral artery due to malalignment of the atlas vertebra may correlate with increased BP and worsening of hypertension. ${ }^{7}$

Research done by Coffee et al and Nicholas et al using MRI techniques showed important correlation between compression of the vertebral artery in subgroup of hypertensive population, but not in normotensives. ${ }^{7,16,20}$ Akimura et al done comparison of the ventrolateral medulla, vertebral arteries, and branches using MRI in hypertensive individuals and control group. They found that out of 32 cases of EHTN, 90.6\% showed compromised neurovascular structures, which were statistically significant. ${ }^{18}$

A study by Jannetta et al showed compressed vascular structures of the left medulla and vagal nerve could be a factor in the development of neurogenic hypertension. Such vascular abnormalities could cause hyperactive autonomic dysfunction of the cardiorespiratory center located in the medulla and affect the left vagus nerve. ${ }^{17}$

Jannetta et al and Levy et al concluded that few hypertensive individuals get notable alleviation of their raised BP after microvascular decompression. ${ }^{17,21}$ This proves the significant role of the vascular structures around the cervical area in maintaining the BP.

\section{Muscular Mechanism}

In FHP, line of gravity passes anteriorly in compared with the normal head neck posture. This causes an increase in the flexion moment. To neutralize this greater external flexion moment and uphold the head in its normal alignment, there is constant isometric contraction of cervical extensor muscles. ${ }^{12}$

Although neural connections have not been identified, it has been proposed that sensory inputs from cervical muscles control cardiorespiratory center, as components of postural reflex. It is proposed that the cervical muscle spindle afferents send signals to the intermedius nucleus of the medulla (InM). The InM has neurochemically different neurons, which send both excitatory and inhibitory extensions to nucleus tractus solitarius (NTS). These data support a new network that may influence possible reflex changes in autonomic variables after cervical muscle spindle afferent stimulation. Cardiorespiratory adjustments can also be induced by the activation of cervical muscle afferents, which can assist changes in cardiorespiratory outflow in preparation for a change in posture. However, the neuronal routes that link these afferent signals to cardiorespiratory control are unidentified. ${ }^{22}$

Edwards et al found a direct neuronal pathway between the cervical muscles and the NTS, the part of the brain that has significant contribution in controlling heart rate and BP. Therefore, any cervical injury or strain of bad posture may lead to trigger responses leading to elevation in BP. The sensory inputs from cervical muscles are forwarded to dorsal horn and the central cervical nucleus of the cervical spinal cord. There is also a substantial cervical muscle afferent extension to the medulla oblongata where fibers end in the external cuneate nucleus and the InM, but this nucleus remains unidentified in the brainstem. ${ }^{22}$ Edwards et al concluded that the InM receives afferent inputs from cervical muscles. The stimulation of cervical muscle spindle afferents as a result of neck flexion can trigger an upsurge in muscle sympathetic nerve activity and arterial BP. These alterations may begin from the cervical extensor musculature, with activation of nerves originating from these muscles. This "cervico-sympathetic reflex" arises from muscle spindles in the cervical extensor muscles..$^{22}$

As suboccipital muscles contain large number of muscle spindles, it is possible that these muscles are also involved in cervicosympathetic reflex. Change in the length of fibers of the suboccipital muscles by chiropractic manipulations of the $\mathrm{C}_{1}$ vertebra may cause change in the BP. This yields supplementary evidence for the involvement of the suboccipital muscles in the cervicosympathetic reflex. The NTS is an important integratory area of autonomic control system. The extension from the InM to the NTS recognized in research done by Edwards et al, therefore, places it in an ideal position to mediate cardiorespiratory changes due to cervical muscle afferent activation. ${ }^{22}$ 
However, further research is essential to assess the role of cervical spine and neck muscle afferents in cardiorespiratory control and high BP.

\section{Conclusion}

Peripheral arterial BP in individuals with FHP is statistically significantly higher than in individuals without FHP.

\section{Conflict of Interest}

None declared.

\section{References}

1 Kisner C, Colby LA. Therapeutic Exercise: Foundations and Techniques. 5th edition. New Delhi: Jaypee Brothers Medical Publishers Ltd; 2007

2 Griegel-Morris P, Larson K, Mueller-Klaus K, Oatis CA. Incidence of common postural abnormalities in the cervical, shoulder, and thoracic regions and their association with pain in two age groups of healthy subjects. Phys Ther 1992;72(6):425-431

3 Brunton J, Brunton E. Reliability of Measuring Natural Head Posture Using the Craniovertebral Angle. Irish Ergonomics Society Annual Conference 2003;37-41

4 Fernández-de-las-Peñas C, Alonso-Blanco C, Cuadrado ML, Pareja JA. Forward head posture and neck mobility in chronic tension-type headache: a blinded, controlled study. Cephalalgia 2006;26(3):314-319

5 Shah SN. Indian guidelines on hypertension-III. JAPI 2013;61(2):1-38

6 Magee DJ. Orthopedic Physical Assessment. 5th edition. New Delhi: Elsevier; 2008

7 Bakris G, Dickholtz M Sr, Meyer PM, et al. Atlas vertebra realignment and achievement of arterial pressure goal in hypertensive patients: a pilot study. J Hum Hypertens 2007;21(5):347-352

8 Thompson W, Ed. ACSM's Guidelines for Exercise Testing and Prescription. 8th edition; 2009

9 Bakris GL, Black HR. Prevention, detection, evaluation and treatment of high blood pressure. JNC-7 2004;1-104. Available at: https://www.nhlbi.nih.gov/files/docs/guidelines/jnc7full. pdf. Accessed November 13, 2019
10 van Niekerk SM, Louw Q Vaughan C, Grimmer-Somers K, Schreve K. Photographic measurement of upper-body sitting posture of high school students: a reliability and validity study. BMC Musculoskelet Disord 2008;9:113

11 Specht G. Specht Physical Therapy - Orthopedic \& Sports Physical Therapy Center, [homepage on the Internet]. 2006. Available at: http://www.spechtpt.com/about_SpechtPT.php. Accessed October 6, 2013

12 Levangie PK, Norkins CC. Joint Structure and Function: A Comprehensive Analysis. 4th edition. New Delhi: Jaypee Brothers Medical Publishers Ltd; 2006.

13 Kapandji IA. The Physiology of Joints. 2nd edition. New York: Churchill Livingstone; 1974

14 Guyton AC, Hall J. Textbook of Medical Physiology. 11th edition. Philadelphia, PA: Saunders; 2006

15 Reis DJ, Morrison S, Ruggiero DA. The C1 area of the brainstem in tonic and reflex control of blood pressure. State of the art lecture. Hypertension 1988;11(2 Pt 2):I8-I13

16 Coffee RE, Nicholas JS, Egan BM, Rumboldt Z, D'Agostino S, Patel SJ. Arterial compression of the retro-olivary sulcus of the medulla in essential hypertension: a multivariate analysis. J Hypertens 2005;23(11):2027-2031

17 Jannetta PJ, Segal R, Wolfson SK Jr. Neurogenic hypertension: etiology and surgical treatment. I. Observations in 53 patients. Ann Surg 1985;201(3):391-398

18 Akimura T, Furutani Y, Jimi Y, et al. Essential hypertension and neurovascular compression at the ventrolateral medulla oblongata: MR evaluation. AJNR Am J Neuroradiol 1995;16(2):401-405

19 Schobel HP, Frank H, Naraghi R, Geiger H, Titz E, Heusser K. Hypertension in patients with neurovascular compression is associated with increased central sympathetic outflow. J Am Soc Nephrol 2002;13(1):35-41

20 Nicholas JS, D'Agostino SJ, Patel SJ. Arterial compression of the retro-olivary sulcus of the ventrolateral medulla in essential hypertension and diabetes. Hypertension 2005;46(4):982-985

21 Levy EI, Scarrow AM, Jannetta PJ. Microvascular decompression in the treatment of hypertension: review and update. Surg Neurol 2001;55(1):2-10, discussion 10-11

22 Edwards IJ, Dallas ML, Poole SL, et al. The neurochemically diverse intermedius nucleus of the medulla as a source of excitatory and inhibitory synaptic input to the nucleus tractus solitarii. J Neurosci 2007;27(31):8324-8333 